\title{
Article \\ Exploring the Decisional Drivers of Deviance: A Qualitative Study of Institutionalized Adolescents in Malaysia
}

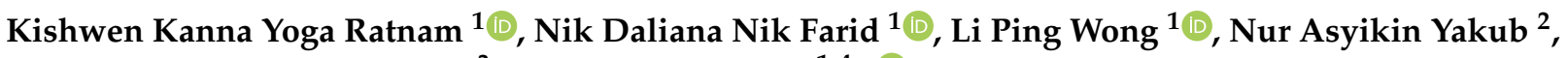 \\ Mohd Alif Idham Abd Hamid ${ }^{3}$ and Maznah Dahlui $1,4, * \mathbb{D}$
}

1 Centre of Population Health, Department of Social and Preventive Medicine, Faculty of Medicine, University of Malaya, Kuala Lumpur 50603, Malaysia; kishwen87@gmail.com (K.K.Y.R.); daliana@ummc.edu.my (N.D.N.F.); wonglp@ummc.edu.my (L.P.W.)

2 Psychology and Human Well-Being Centre, Faculty of Social Sciences and Humanities, National University of Malaysia, Bangi 43600, Malaysia; nurasyikin6666@ummc.edu.my

3 Julius Centre, Faculty of Medicine, University of Malaya, Kuala Lumpur 50603, Malaysia; alifidham@um.edu.my

4 Faculty of Public Health, Universitas Airlangga, Surabaya 60115, Indonesia

* Correspondence: maznahd@ummc.edu.my

check for updates

Citation: Yoga Ratnam, K.K.; Nik

Farid, N.D.; Wong, L.P.; Yakub, N.A.; Abd Hamid, M.A.I.; Dahlui, M. Exploring the Decisional Drivers of Deviance: A Qualitative Study of Institutionalized Adolescents in Malaysia. Adolescents 2022, 2, 86-100. https://doi.org/10.3390/ adolescents2010009

Academic Editors: Christiane Stock and Marjan Mohammadzadeh

Received: 10 November 2021

Accepted: 14 February 2022

Published: 23 February 2022

Publisher's Note: MDPI stays neutral with regard to jurisdictional claims in published maps and institutional affiliations.

Copyright: (c) 2022 by the authors. Licensee MDPI, Basel, Switzerland. This article is an open access article distributed under the terms and conditions of the Creative Commons Attribution (CC BY) license (https:// creativecommons.org/licenses/by/ $4.0 /)$.

\begin{abstract}
Adolescents who break the law or deviate from social norms are often criminalized as their behavior is perceived as resulting from conscious choices. However, it is likely that such choices result from their circumstances, namely the failure of their proximal environment to safeguard their wellbeing. The purpose of this study was to gain an in-depth understanding of the background variables and choices that led these institutionalized adolescents down the road of deviance. We conducted a qualitative study using semi-structured in-depth interviews, conducted between March and May 2018, with 18 participants aged between 15 to 19 years. Participants were purposefully sampled from various welfare institutions in the states of Kuala Lumpur and Selangor, Malaysia. Audio recordings of interviews were transcribed verbatim and analyzed thematically. We found three core themes, (i) Sources of Distress, (ii) Drivers of Deviance, and (iii) Adjustment Strategies for Coping with Distress, which appeared to have influenced participants' life trajectories and eventual institutionalization. The findings showed that participants were disadvantaged by backgrounds of risk and vulnerability, characterized by a lack of social support and opportunities for personal development, negative schooling experiences, and negative peer interactions. Substance abuse, which provided a coping strategy for the participants, may have further contributed to their delinquency. Drawing upon the socio ecological model (SEM), we systematically identify interventional opportunities at the individual, community, and policy levels to safeguard the wellbeing of at-risk adolescents. We make recommendations aimed at improving the family dynamics, promoting a healthy schooling experience, and transforming neighborhoods into a safe and nurturing environment.
\end{abstract}

Keywords: delinquency; youth; institutionalized; juvenile; Malaysia

\section{Introduction}

Globally, deviance remains a public health concern. It is a pervasive social phenomenon that threatens the peace of a community and productivity of a country [1]. The scale of the problem is reflected by the significant worldwide prevalence of adolescents who are in conflict with the law and currently institutionalized.

The understanding, or definition, of delinquency varies from one society or country to another as a result of cultural and historical factors, as well as traditional religious laws. Legally, 'juvenile delinquent' is a term used to refer to a minor who has committed an illegal act [2] that constitutes a criminal [3] and status offenses [4]. According to the Malaysian Ministry of Education, delinquency in school includes violation of both the Penal Code and the school norms as cited in Hussin, 2007. 
As in other parts of the world, Malaysia is seeing rising rates of delinquency among adolescents. In 2013, the Malaysian Ministry of Home Affairs (MOHA) reported 8704 cases involving children or adolescents, a 150\% increase from the previous year [5]. Juvenile deviant behavior is a complex issue linked to multiple factors including peer influence, socioeconomic background, parenting, neighborhood factors, and other precursors such as physical or sexual abuse and violence [6-9]. In addition, research has identified mental health as factor influencing delinquency. A study conducted on juvenile detainees in Malaysia [10] found that $93 \%$ of the 155 participants had, at a minimum, one diagnosable psychiatric disorder, with over a quarter having two or more disorders. Moreover, the Malaysian National Anti-Drug Agency (AADK) 2016 report revealed that out of the total of 30,844 drug users identified, $5.46 \%$ were adolescents aged between 13 and 19 years old [11].

The socio-ecological model proposes that individual, interpersonal, community, organizational, and societal factors have direct and indirect influences on lifestyle, behavior choices, and health [12]. Individual factors that are the highest predictors of delinquency are low intelligence (IQ test scores), poor educational performance, personality, impulsiveness and hyperactivity, and early aggressive behaviors [13]. Furthermore, individuals with a history of offense often had bad school experience due to prejudice. This dissociative feeling of not being able to blend in with their fellow schoolmates causes them to revert back to their delinquent behavior [14].

Interpersonal factors, including family and peers, play a significant role in the development of delinquent behavior among youth. For example, adolescents in Malaysian juvenile rehabilitation centers expressed unhappiness with their parents, citing their experiences of parental/familial violence or strained relationships [15]. Adolescents who come from a poor family structure such as those with a single parent, a neglectful parents, or parents of lower educational level have been associated with substance abuse as driver of delinquency [16]. Meanwhile, males and females reported different types of traumatic family circumstances that resulted in their delinquent behavior. Male adolescents often reported physical abuse, whereas females claimed sexual abuse, to be the cause for their delinquent behavior [17]. Conversely, a cohesive family unit minimizes the chance that a child will be involved in delinquent behavior. A balanced level of cohesiveness and flexibility is favorable to healthy family functioning and greater family satisfaction [18].

Outside the family domain, peer group factors play a large role in determining whether an individual will become involved in delinquent behavior [19-21]. A recent study in Malaysia found peer pressure to be among the factors directly associated with early sexual initiation among 19 adolescents from welfare institutions [20]. Age and educational level are the two main factors that cause adolescents to conform to peer pressure, which leads to delinquent behavior. According to a study, adolescents aged between 12 and 17 years old who were dropouts from school and who had friends involved in substance abuse tend to get involved in substance abuse themselves too. This shows that young adolescents with low educational level are more susceptible to negative peer influence because they lack the knowledge to differentiate what is right and wrong. They tend to follow their peers' footsteps due to pressure under a false impression of being "popular" in the crowd [22].

Beyond the interpersonal domain, the community forms the broader environment for growing youth. Factors in this domain can increase or decrease a youth's likelihood of engaging in delinquency. Neighborhood disadvantage is the absence of settings that encourage healthy child development, such as settings for learning (e.g., libraries), social and recreational activities (e.g., parks), child care, quality schools, health care services, and employment opportunities. These characteristics of neighborhoods and schools have been associated with mental, emotional, and behavioral problems among children [23]. It was found that adolescents living in poorer and smaller residential areas or neighborhoods without beneficial infrastructures had a greater tendency to develop delinquent behavior such as substance abuse and vandalism because they are prone to spend more time with their friends wandering around aimlessly to kill boredom [24]. 
An adolescents' experience depends on various factors, with the culture of the local community being one of the crucial components. However, there is a paucity of available data on the experience of institutionalized adolescents in the Malaysian context. Therefore, interventions based on research in other settings might not be effective or practicable in the Malaysian context due to sociocultural differences. This study aims to bridge this gap in the literature by conducting a qualitative study to explore the background variables underpinning institutionalized youths' deviant behavior and their perceptions about the life circumstances that had led to their delinquent behavior. This could be done by analyzing the socio-demographic characteristics of the participants and in-depth interviews with them. Our goal is to generate actionable data to inform the development of strategies to minimize the risk of delinquency among Malaysian youth.

\section{Materials and Methods}

We used a qualitative approach to gain a deeper understanding of institutionalized participants' perspectives and experiences through an interactive process [25] of open-ended questions and probes [26]. We conducted multiple semi-structured in-depth interviews to obtain thick descriptions from participants. Data were collected from March 2018 to May 2018. Figure 1 shows the study design of this investigation.

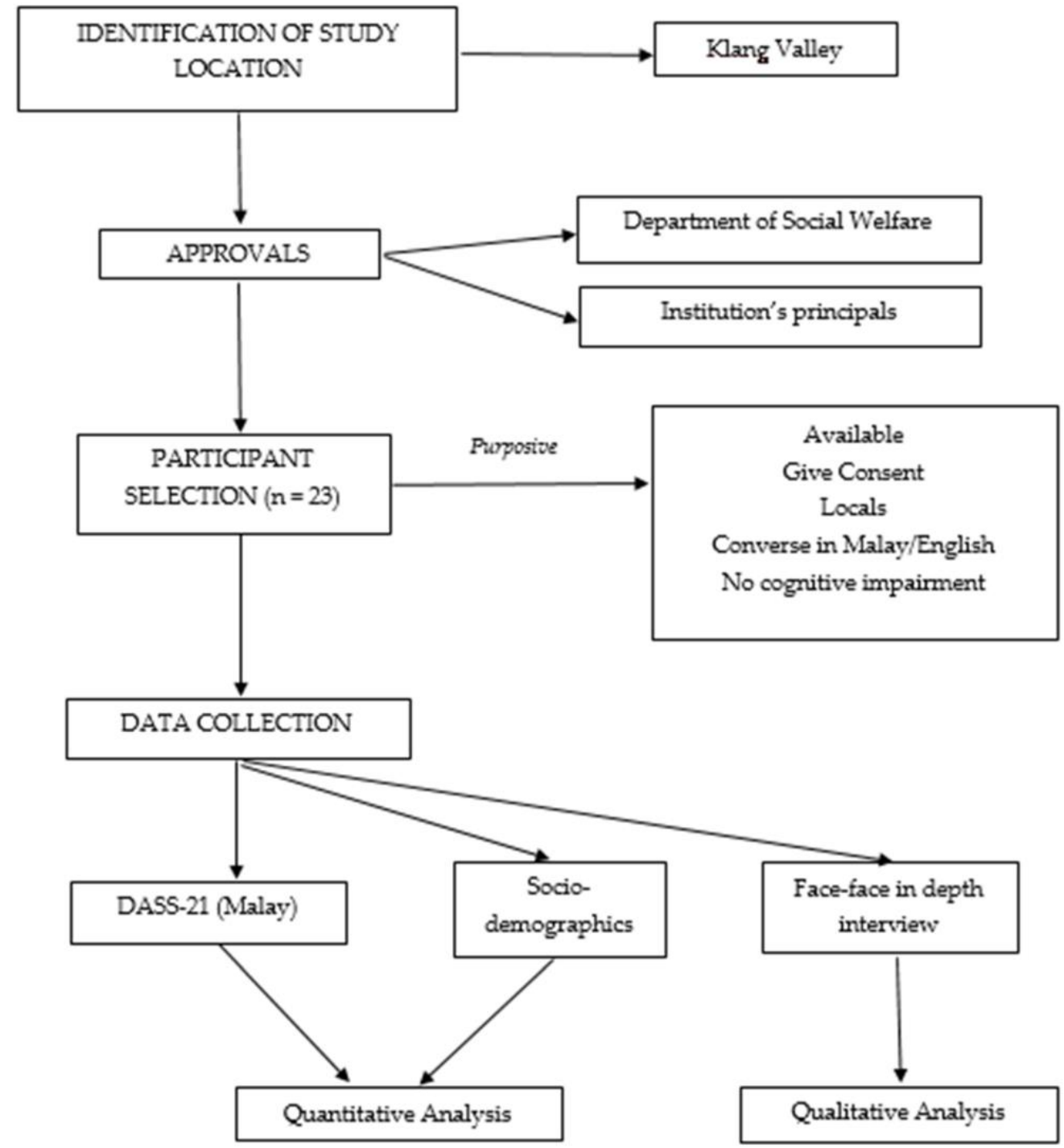

Figure 1. Study design of investigation. 


\subsection{Participants}

The sampling frame encompassed institutionalized adolescents from institutions under the purview of the Department of Social Welfare, Malaysia. These included institutions offering protection, rehabilitation and vocational training services within Peninsular Malaysia. We recruited participants who could converse in Malay or English and were without cognitive impairment. Eighteen adolescents aged between 15 and 19 took part in this study. To capture a broad variation in experiences, we recruited participants from numerous institutions to ensure maximum variation among our respondents [27]. A purposive sampling method allowed us to recruit participants who possessed the desired characteristics for this study [27], which was a history of deviant behavior. Data saturation was taken as the endpoint of participant recruitment, in which we continued purposeful convenience sampling to the point there were no new categories, themes, or descriptions emerging from the data analysis [28].

\subsection{Procedures}

\subsubsection{Data Collection}

Engagement with participants commenced after the study received approval from the Medical Ethics Committee of University of Malaya (Reference Number: UM.TNC2/UMREC199), permission from the Department of Social Welfare, Malaysia (Reference Number JKMM 100/12/5/2: 2018/028) and the heads of the institutions. Primary data were collected via sociodemographic forms and semi-structured in-depth interviews. Participants provided information about their age, gender, ethnicity, residential area, educational level, and family structure by answering a self-administered questionnaire devised by our research team. Experienced researchers with professional qualifications in adolescence studies and psychology took part in the evaluation interviews. They were blinded to these data prior to the interviews to prevent interviewer bias. Pertinent data derived from the interviews were then extracted and combined with details from the self-administered questionnaire to form Table 1.

We conducted the interviews in a private room within institution grounds. First, we briefed potential participants about the study and gave them the Participant Information Sheet before obtaining informed consent from the participants and the institution principals, who acted as their legal guardians. Throughout the study, there were neither any refusals nor withdrawals. We began each interview with a casual chat to build rapport and put the participant at ease. We audio-recorded the interviews, and one researcher took written notes. Interviews lasted between 30 and $45 \mathrm{~min}$. Interviewers used a piloted topic guide that drew upon the SEM to ensure that conversations covered the various factors that have the potential to influence an individual's behavior and state of being. To achieve this aim, the questions were designed to elicit participants' experiential narratives, mainly focusing on the individual, family, peer, and community domains. To encourage disclosure, we initiated new topics, largely with open-ended questions such as "Could you please take me through the events that preceded your institutionalization?" and "How did you spend your free time before being institutionalized"? In addition, we assigned pseudonyms to participants and removed descriptors that identified the institution.

Considering the vulnerable participant group and the possibility that the intrusive nature of the study could invoke suppressed traumatic memories, we took care to remain sensitive to the participants' verbal and non-verbal cues that indicated emotional discomfort during the interviews. We established referral mechanisms in case participants disclosed or exhibited signs of psychological disturbance. The participant would be referred to the in-house counsellor for a review, who would subsequently escalate the case to the nearest government health facility with psychiatric services should specialist consultation be required. 
Table 1. Socio-demographic Characteristics of Participants.

\begin{tabular}{|c|c|c|}
\hline Socio-Demographic Characteristics & Frequency $(n)$ & Percentage $(\%)$ \\
\hline \multicolumn{3}{|l|}{ Age (in years) } \\
\hline $16-17$ & 7 & 38.9 \\
\hline $18-19$ & 11 & 61.1 \\
\hline \multicolumn{3}{|l|}{ Gender } \\
\hline Male & 11 & 61.1 \\
\hline Female & 7 & 38.9 \\
\hline \multicolumn{3}{|l|}{ Ethnicity } \\
\hline Malay & 18 & 100 \\
\hline Others & 0 & 0 \\
\hline \multicolumn{3}{|l|}{ Educational level } \\
\hline Completed secondary education & 5 & 27.8 \\
\hline Still schooling & 4 & 22.2 \\
\hline $\begin{array}{l}\text { Did not complete secondary } \\
\text { education }\end{array}$ & 9 & 50.0 \\
\hline \multicolumn{3}{|l|}{ Family structure } \\
\hline Two biological parents & 5 & 27.8 \\
\hline Single parent & 9 & 50.0 \\
\hline Both parents deceased & 4 & 22.2 \\
\hline \multicolumn{3}{|l|}{ Residential area } \\
\hline Rural & 5 & 27.8 \\
\hline Sub-urban & 12 & 66.7 \\
\hline Urban & 1 & 5.5 \\
\hline \multicolumn{3}{|l|}{ History of drug use } \\
\hline Yes & 10 & 55.6 \\
\hline No & 8 & 44.4 \\
\hline \multicolumn{3}{|l|}{ History of sexual intercourse } \\
\hline Yes & 7 & 38.9 \\
\hline No & 11 & 61.1 \\
\hline \multicolumn{3}{|l|}{ Duration of institutionalization } \\
\hline Less than 6 months & 2 & 11.1 \\
\hline More than 6 months & 16 & 88.9 \\
\hline Total & 18 & 100 \\
\hline
\end{tabular}

\subsubsection{Data Analysis}

We transcribed the recorded interviews verbatim in Malay, with two members of the research team working independently to achieve triangulation of the researchers. However, for the purpose of publication and sharing of findings, we translated selected excerpts and quotes into English.

Recognizing the central role a researcher plays in in qualitative studies [29], throughout the research process, we endeavored to avoid making a priori assumptions based on previous reading, while drawing on reading of relevant literature to remain sensitive to the subtler features of the raw data [30].

We employed the color coding technique to highlight meaningful text within the narratives. We then organized them into themes according to the concepts identified in the socioecological framework. We were also careful not to ignore findings unexpectedly emerging from the data [31]. As an initiative to avoid researcher bias and to strengthen rigor, each step of the qualitative analysis process was conducted independently by the researcher and another team member to achieve triangulation of the coding process. Additionally, Cohen's kappa coefficient was calculated from the coding outcome of 5 adolescents to quantitively evaluate the inter-rater agreement between the respondents.

We then compared and discussed the findings and tabulated the refined findings in a master table of themes. The entire team then discussed the findings for validation before they were finalized. These discussions were done in order to achieve triangulation of the theoretical interpretation. 
Chi-square analysis was performed to evaluate the correlation between socio-demographic characteristics of the respondents and the themes identified. Confounding factors that might influence the theme identified among these youths were controlled via multivariate regression analysis.

\section{Results}

The finalized analysis comprised three themes and twelve sub-themes (Table 2) that were believed to have contributed to participants' delinquent experience. The three overarching themes were "Sources of Distress," "Drivers of Delinquency," and "Adjustment Strategies for Coping with Distress". Cohen's kappa coefficient for the agreement of coding outcome for five adolescents was 0.98 among three researchers.

Table 2. Themes of study.

\begin{tabular}{|c|c|c|c|c|}
\hline \multicolumn{5}{|c|}{ Main Themes } \\
\hline \multicolumn{2}{|r|}{ Sources of Distress } & \multicolumn{2}{|r|}{ Drivers of Delinquency } & Adjustment Strategies for Coping with Distress \\
\hline \multicolumn{5}{|c|}{ Subthemes } \\
\hline i. & Traumatic family circumstances & i. & Inadequate family support & Substance abuse \\
\hline ii. & Damaging peer interactions & ii. & Laxity in supervision & \\
\hline iii. & Internalizing domestic difficulties & iii. & Peer influence & \\
\hline iv. & Pressure to conform & iv. & Neighborhood nuisance & \\
\hline & & v. & Negative school experience & \\
\hline & & vi. & Boredom & \\
\hline & & vii. & Social media and sexual initiation & \\
\hline
\end{tabular}

\subsection{Theme 1: Sources of Distress}

Almost all the participants admitted to having faced adversities in different aspects of their lives, be it at home, at school, or within the community at large. The emotional turmoil experienced as a result of these adversities increased their vulnerability and was a major distraction in their pursuit of living their lives to their fullest potential. The main sources of distress were family circumstances, domestic difficulties, peer interactions, and pressure to conform.

\subsubsection{Traumatic Family Circumstances}

A number of participants mentioned experiencing traumatic family circumstances in their childhood. Chi-square analysis, performed to evaluate the correlation between socio-demographic characteristics of the respondents and traumatic family circumstances, revealed this theme to be significantly correlated with Education Level $(p=0.045)$ and Family Structure $(p=0.045)$. However, multivariate regression analysis revealed that neither Education Level nor Family Structure is an independent predictor of Traumatic Family Circumstances.

An example of traumatic family circumstances is what has been revealed by Lanisa (female, 18), who witnessed her parents' constant arguments, which eventually led to their divorce when she was ten. She revealed that she had been physically abused many times by her father and also vaguely recounted an episode when her father made inappropriate physical contact with her. She said, "I was hit very badly by my father. He hit me and my sister as though he wanted to kill us. There also was an instance I vaguely remember once he (father) kissed me on my lips".

\subsubsection{Internalizing Domestic Difficulties}

All eighteen participants talked about experiences resulting from their family's financial constraints, for example, rarely enjoying family outings or holidays. This also dampened their spirits in realizing their childhood ambitions. Most said that their fathers worked long hours, with some reporting that their fathers had to hold two jobs to make 
ends meet. From their narratives, it appeared that financial hardships often resulted in tension within the home atmosphere. For example, Ruly (male, 18) described how he would help diffuse his parents' arguments, "My father lost his temper and swung a chair in front of my mother. I was there. I scolded my father. I told him, 'you're an adult, don't do these kinds of things'"'.

It was evident that parental arguments denied these participants the opportunity to receive the love they deserve because their parents were either emotionally drained or too busy sorting out their own domestic issues. Masy (female, 16) confessed that she contemplated suicide. When asked about the reason for this, she explained, "I never got the love and attention that I was yearning for".

The lack of positive support and getting embroiled in family drama affected these teens adversely. Chi-square analysis did not reveal any correlation between this particular theme and the respondent's socio demographic characteristics.

\subsubsection{Damaging Peer Interactions}

Participants revealed an absence of camaraderie, compassion and understanding amongst peers in their school environment. Peer interactions were deemed more detrimental than beneficial to the participants' academic pursuit and personal growth. Masy (female, 16) said, "I was bullied in school by my seniors when I first joined. They told me to wash their clothes, shoes and socks. I felt angry and hatred, everything. Imagine the socks were kept in dirty water for a month, then I was forced to wash it ( ... ) the bad smell ..." (while grimacing). Ruly (male, 18), who grew up in foster care, was expelled from school in Form 3 (age 15) for fighting with peers. He said, "I fought a lot in school. Because initially I was bullied in primary school. So when I entered secondary school, it was my turn to bully others". Participants who said they enjoyed the company of their peers mainly talked about their common interests in delinquent activities such as truancy, smoking, and experimenting with drugs. Damaging peer interaction was found to be significantly correlated to Family Structure $(p=0.030)$ and Duration of Institutionalization $(p=0.034)$ via Chi square analysis. None of these variables were found to be independent predictor of Damaging Peer Interaction via multivariate regression analysis.

\subsubsection{Pressure to Conform}

Experiencing a punitive system in a lifestyle that is already void of fun family trips and bonding time affected participants in a negative way. A few participants mentioned strict parental rules as a source of frustration that fueled their determination to seek pleasure outside the home. More than half the participants described feeling stressed because of expectations to conform to the norms of their environment. Diane (female, 16) talked about her strict parents, saying, "Like ( . . ) in the past when I used to go out often and come home late, my father would be angry. It made me stress. By ten at night I was meant to be home, not doing anything, not holding my handphone. Until I couldn't take it anymore, I ran away from home to find my own freedom". No correlation between this particular theme and the respondent's socio demographic characteristics was found via Chi-square analysis.

\subsection{Theme 2: Drivers of Delinquency}

Seven sub-themes were identified as potential contributors to the participants' delinquent behavior: inadequate parental support, laxity in supervision, peer influence, neighborhood nuisance, experiencing social failure, boredom, and social media and sexual initiation.

\subsubsection{Inadequate Parental Support}

Most of the participants did not describe experiences of parental support, in terms of support for their hobbies, education, and financial, or emotional wellbeing. A few participants mentioned that the disconnect between themselves and their parents caused them to turn to their peers to confide in, which most of the time resulted in receiving bad 
advice. Chi-square analysis did not reveal any correlation between this particular theme with the respondents' sociodemographic characteristics.

Masy (female, 16) shared, “Because even if I confide in my mother, she won't do anything, all she does is just listen and says, 'hah, that's normal, get over it'". Nisha (female, 18) described her experience, saying "Even in the house I have no one to chill with. I mean, my parents are much older than me. They can't relate to my life. Even if I ask them out to go to the karaoke, they would refuse. It's just like that (sighs). I ask my mum to tag along for a movie, she would refuse. But then when I ask their permission to go out on my own, they won't allow me". Similarly, Diane (female, 16) said, "But my mother always has different perspectives, so I feel like it's not best to talk with her. So instead, I talk with my friends. That's how I value my friend's opinions more than my family".

\subsubsection{Laxity in Supervision}

The majority of participants claimed to have had the freedom to hang out with their friends until late at night, or even into early morning, without being answerable to their parents in regard to their whereabouts. Diane (female, 16) shared, "I'm not too sure about their (friends) families. But its weird right, most of my friends who hang out late at night, they can chill out just like that. It's like they don't receive calls from their mothers, never have I noticed that. I found that strange. All my friends ( . . ) their families don't seem to bother if they loiter at night". Only Family Structure $(p=0.031)$ was found to be correlated with the Laxity in Supervision theme via Chi square analysis.

\subsubsection{Peer Influence}

Almost all the participants talked about the role their peers played in their deviant behavior. Their relationship with certain peers encouraged them to utilize unhealthy or negative ways to solve their troubles and keep themselves happy. For example, Lanisa (female, 18) said, "I liked school. But I was influenced by friends. They asked me to skip class, hang out in the toilets, and loiter around. At first I skipped class, but then later on I skipped school entirely". Diane (female, 16) shared her first experience with smoking with some friends, "They used to smoke and they offered me to try. I tried it once, then it felt like it was great". She went on to explain how she was influenced by methamphetamines while she was in Form One. "At first, I just knew that certain types of drugs existed. Then I when I started hanging out late nights and not returning home, I met these boys who use drugs. One day my friend told me 'hey try this (ice) lah'. And that's how I started using it frequently", she said.

The vulnerable status of the participants rendered them easy targets to be recruited into the circle of vice. These deviant peers, who many of the respondents said were either school drop outs or older school mates, capitalized on the participants' tough times to introduce them to delinquent habits. Peer Influence was found to be significantly associated with only Education Level $(p=0.031)$ via Chi square analysis.

\subsubsection{Neighborhood Nuisance}

The literature suggests that a healthy neighborhood facilitates the successful development of a child and is equipped with the right ingredients to promote the growth of the mind, body, and soul [32]. The overwhelming majority of participants grew up in underserved neighborhoods that lacked a cohesive community, security, and avenues for pro-social activities. From their narratives, it was clear that they did not have safe outlets for leisure activities. Moreover, participants were able to interact with school dropouts, unemployed youths, and drug dealers in their community, thus further increasing their exposure to unhealthy activities, such as drugs and alcohol. The presence of these individuals in the neighborhood also reflected the community's tolerance for delinquent behavior.

Many participants said that they had befriended tokans (drug dealers) within the neighborhood, which made drugs easily accessible. For example, Diane (female, 16) shared, "The drugs were supplied by the older kids who hung out in my neighborhood. It was 
easy to get them (drugs) because the dealers were friends with my friends". Interestingly, a correlation between Gender $(p=0.40)$ of the respondent and the presence of Neighborhood Nuisance when evaluated via Chi square analysis. This suggests that neighborhood nuisance may have a different influence on participants depending on their gender.

\subsubsection{Negative School Experience}

The stories shared by participants indicated that educational institutions can be a tough environment for an adolescent to negotiate, especially those who lack social support and are experiencing difficult times. Lanisa (female, 18) talked about her negative experience at school, saying "I was not allowed to sit on the chair in class. I was made to write my work on the floor. Just me alone. That is why I did not like my teacher. The teachers also were not enthusiastic about teaching. And another thing, the classroom environment was very playful. I could not concentrate at all. So I was fed up and did not study lah". In addition, Nisha (female, 18) said, "Honestly, I have mixed feelings about school. I like going to school because I get to meet my friends. But I really do not like the learning part. I feel so sleepy in class. The teachers are just talking nonsense always".

Another participant said that she was frequently picked on by her class monitor, who made false reports against her. Consequently, her class teacher would dispense punishment without performing any prior investigation on the matter. Being publicly embarrassed damaged her self-esteem. Additionally, none of the participants who described experiencing hardship in school expressed their intention to speak with a school counsellor, despite being aware of such services. Chi-square analysis did not reveal any correlation between Negative School Experience and the respondents' socio demographic characteristics.

\subsubsection{Boredom}

It was apparent from all the participants' narratives that they were unable to seek means of catharsis nor to channel their energy constructively. The inability to occupy themselves with constructive activities led their minds to wander and seek avenues to escape the reality of their predicament. Most of them resorted to hanging out with their friends and wasting their time, which facilitated their interaction with other delinquents. Eman (male, 15) said, "My version of a fun time and being happy? I chill out by the highway with my friends. We gather around with our motorbikes and just chill at the usual lay-bys". No correlation between this particular theme and the respondents' socio demographic characteristics was found via Chi-square analysis.

\subsubsection{Social Media and Sexual Initiation}

The narratives showed that possession of a smartphone allowed participants unsupervised access to the outside world. The participants in this study were literally left to their own devices, as their parents and community could not help them occupy their time wisely. All the female participants who had experienced teenage pregnancy cited the use of a social networking site as a medium of communication with their boyfriend at that time. The romantic exchanges online soon led to physical intimacy and ultimately early pregnancy. Diane (female, 16) said the following about how she began relationships with male friends "Whenever we chill out together, they will for sure ask for our account (WeChat) details. After the first meet, when I reach home I will see a friend request on my phone ... that is how it goes. Then, after some chat he will ask me to couple up. Sometimes I just couple up for fun, but it will turn out to be a serious relationship. That is how it all happens". None of the respondents' socio demographic characteristics were correlated to this particular theme when Chi-square analysis was done.

\subsection{Theme 3: Adjustment Strategies for Coping with Distress Substance Abuse}

More than half the participants in this study admitted to having experimented with various types of substances. Nevertheless, no correlation can be concluded between any of 
the respondent's sociodemographic characteristics and Substance Abuse via Chi square analysis. Substance use appeared to be an element that clouded their judgment, eventually leading to addiction and other problematic behaviors. Many of the participants discovered the stress-relieving effects of drugs through their peers and by researching it online via smartphones. The high from drugs provided them with a mechanism to dissociate from their feelings of distress but also led to other delinquent behavior. Talking about how she started using methamphetamines, Masy (female, 16) said, "I really needed something or a way to reduce the stress. So I googled lah ... ways to deal with stress ... Although there were many examples, I chose drugs because it seemed to be an easy solution". Yedam (male, 19) also talked about using drugs as a way to relieve stress, saying "Back then in school, you know (... ) it was kind of normal to feel fed up with the system and how the teachers were and all (... ) Additionally, at that point my friends ask me if I wanted some 'medicine' to reduce the stress ( ... ) so I ended up using it (marijuana)".

\section{Discussion}

The findings of this study indicate that five major factors played a role in participants' experiences that led to their institutionalization for deviant behavior (Figure 2). As the model shows, deviance is a product of an interplay of circumstances that originates within the proximal environment of an adolescent.

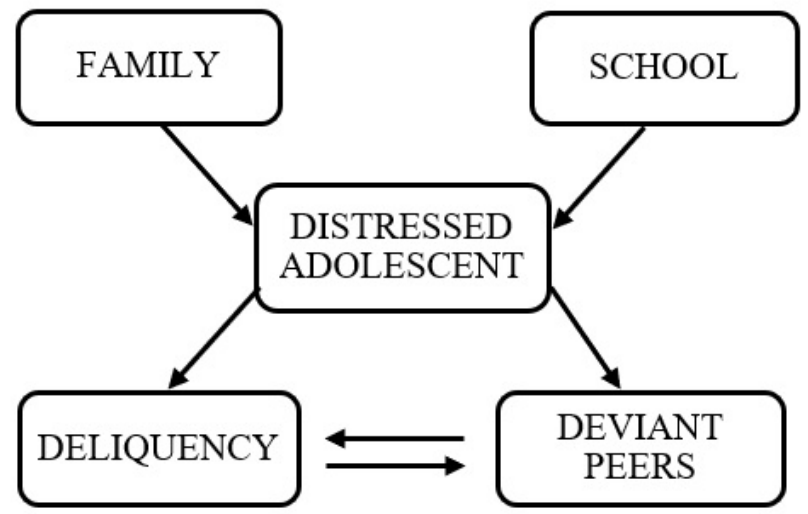

Figure 2. Five major factors leading to institutionalization.

Dysfunctional family dynamics seemed to be a prevalent circumstance in the lives of the participants, which is consistent with the literature in Malaysia [33] and in other countries [34]. As participants' narrative showed, when they did not receive support from their family members, they turned to their peers, who often offered unhealthy solutions. The findings of this study underscore the importance of the home environment and family ties in shaping the life trajectories of the participants. This is consistent with results from another local study, which stated that juvenile detainees had strained relationships with their parents and harbored resentment towards them for various reasons such as divorce and neglect [15].

Adolescents in this study related unfortunate experiences at school, including being bullied by peers and punished by their teachers. The lack of positive relationships in their school environment dampened their interest in learning. Compounded by the lack of social support, this led the participants to rebel against the school system and move towards delinquent behavior and peers. The findings from our study urge teachers to consider that a student's misbehavior may not always be an act of insolence but could belie deeply rooted psychological problems that require serious attention. For instance, low achievement in school and lack of ability to foresee the potential consequences of committing an offense can be associated with personality traits including hyperactivity, impulsiveness, and restlessness [6]. 
Peers emerged as a crucial interpersonal element in the pathway to delinquency. We found that all the participants had experienced negative peer influences prior to institutionalization. Participants had gradually become accustomed to the delinquent lifestyle, subsequently committing offenses that led to their remand. Peer interactions were also closely linked to substance use. As has been found in other settings [4], substance use was common among the participants, with more than half reporting the use of illegal drugs. It is important to note the revelation of the reciprocal relationship between deviant peer association and substance abuse. As the model shows, once participants had come into contact with deviant peers, they were introduced into delinquent behaviors such as substance abuse. Participants' involvement in delinquent behavior led to new friendships with peers who reinforced and sustained delinquent habits. However, an additional finding from the interviews is that within an institutional setting, peer influence led to positive effects. Participants talked about fostering emphatic relationships and receiving support from other residents at their facility, which made their period of institutionalization more bearable and, to a certain extent, enjoyable. Further research will be needed to understand the dynamics of peer behavior in different social settings.

In line with findings from studies in other countries (e.g., Chung and Steinberg, 2006) [32], our study unveiled how neighborhood compositional features enable deviant behavior. This supports previous findings linking economically disadvantaged neighborhoods with mental, emotional, and behavioral problems among children [23]. Like other teenagers, the participants' lives largely revolved around school and their neighborhoods. However, living in underserved neighborhoods meant limited pro-social activities and opportunities to participate in productive activities. The lack of public amenities and infrastructure within the housing area inadvertently led the study participants to gravitate towards activities such as loitering with friends and using drugs to distract them from their mundane lifestyles.

The findings showed that social media served as an interactive platform that facilitated some of the participants' deviant behavior. Having a smartphone enabled participants to forge social relationships without parental supervision or consent. The narratives of the female participants showcased how socializing online quickly progressed to sexual activity offline. This is a cause for concern because early sexual initiation gives rise to a myriad of public health issues [35].

\section{Strengths and Limitations}

Our use of a qualitative approach enabled the uncovering of sensitive and detailed information, which would likely have not been obtained if we had employed standardized instruments with pre-determined responses [36]. We were able to elicit first-hand information about what participants had faced in their lives that led them into institutional care. We explored each participant's experiences, life circumstances, choices, and critical reflections at a personal level [37]. With the goal of producing a trustworthy study, we ensured credibility through the prolonged engagement with the subject matter and investigator triangulation [38]. During the process of data collection and analysis, the principal investigator was privileged to be working alongside a clinical psychologist and two senior public health physicians who possessed first-hand experience in research involving adolescents. The collective strength and diverse insights within our research team promoted a broader perspective in the interpretation of the interview findings, thus preventing researcher bias [39]. To address the issues of dependability and confirmability, we established an audit trail of translated transcripts, related documents for data analysis, and team discussions. We kept detailed records of the processes and procedures of the groundwork and fieldwork involved in operationalizing our study protocol [38].

Although this study has several strengths, there are some limitations that should be noted. Firstly, respondents of Malay ethnicity were overrepresented in this study. The reason for such a sample composition was that adolescents of Malay ethnicity composed the overwhelming majority of detainees in all the institutions during the data collection. 
The research team did request the institution heads for a diverse sample; however, the recruitment was dependent on the availability of participants due to their strict schedules. Secondly, this study recruited participants only from welfare institutions. We recommend that future studies include institutions under the Prisons Department to garner a more diverse sampling frame, enabling a broader exploration of delinquency. In addition, extending the scope by respondents to include relevant stakeholders such as social workers, police officers, teachers, and parents will provide further better understand with regard to the background variables influencing adolescents' involvement in delinquency. Furthermore, due to the limited number of participants included in the study, statistical comparison between the socio-demographic factors could not be performed. Thus, whether the themes vary according to differences in socio-demographic factors could not be determined. Since this study mainly focused on the qualitative aspect of research, the rate of agreement between researchers was not assessed. Information extracted from interviews was merely discussed among the researchers, and any disagreement was just resolved in meetings. This approach may pose certain degree of researcher bias that could affect the outcome of the study. Lastly, information including recurrence of delinquent offence, participants' mental health state and emotional stability were also not recorded or evaluated in the current study.

\section{Conclusions and Recommendations}

In conclusion, our findings show that three themes have contributed to the delinquent experience in adolescents. The three themes include sources of distress (traumatic family circumstances, damaging peer interactions, internalizing domestic difficulties, and pressure to conform), drivers of delinquency (inadequate family support, laxity in supervision, peer influence, neighborhood nuisance, negative school experience, boredom, social media, and sexual initiation), and adjustment strategies for coping with distress (substance abuse). These themes are closely related with the five major factors, namely family, school, delinquency, and deviant peers, that play a role in adolescent's experience that could lead to their institutionalization for deviant behavior.

Understanding these contributing factors would help with the creation of some recommendations to reduce delinquency among adolescents. For instance, insight into the lifestyle and perspectives of institutionalized youth can help inform delinquency prevention policy and mental health promotion. We believe that the findings of our study are transferrable to the population of at-risk youth and can be translated into interventional strategies that are culturally tailored to the Malaysian context. Firstly, collaborations between schools and various governmental agencies must be established, or improved, to better benefit teachers and students. Anti-bullying campaigns and anti-drug programs will be a good start in addressing the social issues arising from delinquency. Similar campaigns can also help minimize risk factors outside the school domain. For example, campaigns about sexual and reproductive health, anti-smoking, and drug abuse could inform youths on the dangers and consequences of indulging in such behavior. Recruiting youths as volunteers in these campaigns not only instils a sense of responsibility but also nurtures them into agents of positive peer influence and ensures the sustainability of the program.

Boredom was portrayed as the precursor for deviant habits among our study participants. Therefore, it may be worthwhile to incentivize attendance at extracurricular sports activities at schools [40] in underserved communities, in addition to organizing activities such as field trips and vocational training classes. This will facilitate the development of hobbies, spark learning interests, and forge healthy peer relationships [41]. The availability of leisure activities for youth in economically disadvantaged communities will offer them an accessible and healthier alternative to unproductive behavior.

Finally, our findings suggest that institutionalized participants have experienced stressful situations in the past, which carries the risk of negatively affecting psychological wellbeing. A large number of studies have consistently reported a high prevalence of mental health problems among youths in institutions $[42,43]$. It has been found that young 
people show high levels of distress and acts of misconduct during their first month of institutionalization, even though they also display attempts to make positive efforts to cope [44]. The findings from these various studies and our current study underscore the need to provide services that can effectively address youth mental health and behavioral problems in institutional care centers. Thus, we recommend periodic mental health screenings be conducted and mental health services made available in all youth welfare institutions. In addition, further research is required to verify results and establish causal relationships between factors leading to deviant behavior.

Author Contributions: Conceptualization, K.K.Y.R., N.D.N.F., L.P.W., N.A.Y., M.A.I.A.H. and M.D.; methodology, K.K.Y.R., N.D.N.F., L.P.W., N.A.Y., M.A.I.A.H. and M.D.; validation, N.D.N.F., L.P.W. and M.D.; formal analysis, K.K.Y.R., N.A.Y., M.A.I.A.H.; investigation, K.K.Y.R., N.A.Y., M.A.I.A.H.; resources, N.D.N.F. and M.D.; data curation, K.K.Y.R., N.A.Y., M.A.I.A.H.; writing一original draft preparation, K.K.Y.R.; writing-review and editing, N.D.N.F., L.P.W., N.A.Y., M.A.I.A.H. and M.D.; visualization, K.K.Y.R.; supervision, N.D.N.F., L.P.W. and M.D.; project administration, M.D.; funding acquisition, M.D. All authors have read and agreed to the published version of the manuscript.

Funding: This research received no external funding.

Institutional Review Board Statement: All study procedures were done in accordance with the standards of the Medical Ethics Committee of the University of Malaya Medical Centre, Kuala Lumpur, Malaysia (Reference Number: UM.TNC2/UMREC-199), and with the 1964 Helsinki declaration and its later amendments or comparable ethical standards. Permission to collect the data was also granted by the Social Welfare Department of Malaysia after the study protocol was reviewed.

Informed Consent Statement: Informed consent was obtained from all participants included in this study.

Data Availability Statement: Not applicable.

Acknowledgments: Not applicable.

Conflicts of Interest: The authors declare no conflict of interest.

\section{References}

1. Adams, M.S.; Evans, T.D. Teacher disapproval, delinquent peers, and self-reported delinquency: A longitudinal test of labeling theory. Urban Rev. 1996, 28, 199-211. [CrossRef]

2. Brown, S.E.; Siegel, L.J.; Senna, J.J. Juvenile Delinquency: Theory, Practice, and Law. Teach. Sociol. 1984, 11, 471. [CrossRef]

3. Young, S.; Greer, B.; Church, R. Juvenile delinquency, welfare, justice and therapeutic interventions: A global perspective. BJPsych Bull. 2017, 41, 21-29. [CrossRef]

4. National Center on Addiction Substance Abuse at Columbia University. Criminal Neglect: Substance Abuse, Juvenile Justice and the Children Left Behind; National Center on Addiction Substance Abuse at Columbia University: New York, NY, USA, 2004. Available online: https:/ / www.ojp.gov/ncjrs/virtual-library/abstracts/criminal-neglect-substance-abuse-juvenile-justice-andchildren-left (accessed on 18 February 2022).

5. Shong, T.S.; Abu Bakar, S.H.; Islam, M.R. Poverty and delinquency: A qualitative study on selected juvenile offenders in Malaysia. Int. Soc. Work 2019, 62, 965-979. [CrossRef]

6. Hyde, J. The Mental Health Crisis in our Juvenile Detention Centers-Shared Justice. 2016. Available online: https:// www.sharedjustice.org/domestic-justice/2016/8/8/the-mental-health-crisis-in-our-juvenile-detention-centers (accessed on 9 November 2021).

7. Loeber, R.; Farrington, D.P. Young children who commit crime: Epidemiology, developmental origins, risk factors, early interventions, and policy implications. Dev. Psychopathol. 2000, 12, 737-762. [CrossRef]

8. Ortega-Campos, E.; García-García, J.; Gil-Fenoy, M.J.; Zaldívar-Basurto, F. Identifying Risk and Protective Factors in Recidivist Juvenile Offenders: A Decision Tree Approach. PLoS ONE 2016, 11, e0160423. [CrossRef]

9. Pinto, A.C.S.; Luna, I.T.; Sivla, A.D.A.; Pinheiro, P.N.D.C.; Braga, V.A.B.; E Souza, A.M.A. Risk factors associated with mental health issues in adolescents: A integrative review. Rev. Esc. Enferm. USP 2014, 48, 555-564. [CrossRef]

10. Aida, S.; Aili, H.; Manveen, K.; Salwina, W.; Subash, K.; Ng, C.; Muhsin, A. Prevalence of psychiatric disorders among juvenile offenders in Malaysian prisons and association with socio-demographic and personal factors. Int. J. Prison. Health 2014, 10, 132-143. [CrossRef]

11. (AADK) NADA. Maklumat Dadah 2013 (Drug Statistics 2013). Ministry of Home Affairs, Malaysia. 2014. Available online: https://www.adk.gov.my/orang-awam/statistik-dadah/?lang=en (accessed on 25 October 2021). 
12. Centres for Disease Control and Prevention. The Social-Ecological Model: A Framework for Prevention; Centres for Disease Control and Prevention: Atlanta, GA, USA, 2018. Available online: https://www.cdc.gov/violenceprevention/overview/socialecologicalmodel.html (accessed on 25 October 2021).

13. Farrington, D.; Welsh, B.C. Saving Children from a Life of Crime: Early Risk Factors and Effective Interventions; Oxford University: Oxford, UK, 2006. [CrossRef]

14. Sulaiman, S.N.; Zainuddin, A. Social Determinants of Drug Abuse Among Youth in Selangor: A Case Study in Serendah's Rehabilitation Centre. J. Adm. Sci. 2021, 18, 219-236.

15. Kuthoos, H.M.; Endut, N.; Selamat, N.H.; Hashim, I.H.; Azmawati, A.A. Juveniles and Their Parents: Narratives of Male \& Female Adolescents in Rehabilitation Centres. World Appl. Sci. J. 2016, 34, 1834-1839.

16. Dube, S.R.; Felitti, V.J.; Dong, M.; Chapman, D.P.; Giles, W.H.; Anda, R.F. Childhood Abuse, Neglect, and Household Dysfunction and the Risk of Illicit Drug Use: The Adverse Childhood Experiences Study. Pediatrics 2003, 111, 564-572. [CrossRef]

17. Kort-Butler, L.A. Coping Styles and Sex Differences in Depressive Symptoms and Delinquent Behavior. J. Youth Adolesc. 2008, 38, 122-136. [CrossRef]

18. Olson, S.L.; Lopez-Duran, N.; Lunkenheimer, E.S.; Chang, H.; Sameroff, A.J. Individual differences in the development of early peer aggression: Integrating contributions of self-regulation, theory of mind, and parenting. Dev. Psychopathol. 2011, 23, 253-266. [CrossRef]

19. Mayes, L.C.; Suchman, N.E. Developmental Pathways to Substance Abuse. In Developmental Psychopathology, 2nd ed.; Wiley: Hoboken, NJ, USA, 2015; pp. 599-619.

20. Nik Farid, N.; Dahlui, M.; Che'Rus, S.; Aziz, N.; Al-Sadat, N. Early Sexual Initiation among Malaysian Adolescents in Welfare Institutions: A Qualitative Study. Arts Soc. Sci. J. 2015, 6, 1-6. [CrossRef]

21. O'Connell, M.E.; Boat, T.; Warner, K.E. Preventing Mental, Emotional, and Behavioral Disorders among Young People: Progress and Possibilities; National Academies Press: Washington, DC, USA, 2009.

22. Wagner, K.D.; Ritt-Olson, A.; Soto, D.W.; Unger, J. Variation in Family Structure Among Urban Adolescents and Its Effects on Drug Use. Subst. Use Misuse 2008, 43, 936-951. [CrossRef]

23. Gershoff, E.T.; Aber, J.L.; Raver, C.C. Child Poverty in the United States: An Evidence-Based Conceptual Framework for Programs and Policies. In Handbook of Applied Developmental Science: Promoting Positive Child, Adolescent, and Family Development Through Research, Policies, and Programs; SAGE Publications Ltd.: London, UK, 2018; pp. 81-136.

24. Razali, A.; Madon, Z. Issues and Challenges of Drug Addiction among Students in Malaysia. Adv. Soc. Sci. Res. J. 2016, 3, 77-89.

25. Wong, L. Qualitative research methods in family medicine: What and why? Malays. Fam. Physician 2008, 3, 70.

26. Whittaker, A. Qualitative methods in general practice research: Experience from the Oceanpoint Study. Fam. Pract. 1996, 13, 310-316. [CrossRef]

27. Palinkas, L.A.; Horwitz, S.M.; Green, C.A.; Wisdom, J.P.; Duan, N.; Hoagwood, K. Purposeful Sampling for Qualitative Data Collection and Analysis in Mixed Method Implementation Research. Adm. Policy Ment. Health Ment. Health Serv. Res. 2015, 42, 533-544. [CrossRef]

28. Guest, G.; Bunce, A.; Johnson, L. How Many Interviews Are Enough? An experiment with data saturation and variability. Field Methods 2006, 18, 59-82. [CrossRef]

29. Walker, S.; Read, S.; Priest, H. Use of reflexivity in a mixed-methods study. Nurse Res. 2013, 20, 38-43. [CrossRef] [PubMed]

30. Tuckett, A.G. Applying thematic analysis theory to practice: A researcher's experience. Contemp. Nurse 2005, 19, 75-87. [CrossRef] [PubMed]

31. Denzin, N.K.; Lincoln, Y.S. The SAGE Handbook of Qualitative Research; Sage: Thousand Oaks, CA, USA, 2018.

32. Chung, H.L.; Steinberg, L. Relations between neighborhood factors, parenting behaviors, peer deviance, and delinquency among serious juvenile offenders. Dev. Psychol. 2006, 42, 319-331. [CrossRef]

33. Abdul Jalal, F.H. Family Functioning and Adolescent Delinquency in Malaysia. Ph.D. Thesis, Iowa State University, Ames, IA, USA, 2005.

34. Troxel, W.M.; Matthews, K.A. What are the costs of marital conflict and dissolution to children's physical health? Clin. Child Fam. Psychol. Rev. 2004, 7, 29-57. [CrossRef] [PubMed]

35. Naidoo, L.; Sewpaul, V. The life experiences of adolescent sexual offenders: Factors that contribute to offending behaviours. Soc. Work. Werk 2014, 50, 84-98. [CrossRef]

36. Ormston, R.; Spencer, L.; Barnard, M.; Snape, D. The foundations of qualitative research. Qual. Res. Pract. A Guide Soc. Sci. Stud. Res. 2014, 2, 52-55.

37. Marshall, C.; Rossman, G.B. Designing Qualitative Research, 3rd ed.; Sage Publications, Inc.: Thousand Oaks, CA, USA, 1999.

38. Shenton, A.K. Strategies for ensuring trustworthiness in qualitative research projects. Educ. Inf. 2004, 22, 63-75. [CrossRef]

39. Korstjens, I.; Moser, A. Series: Practical guidance to qualitative research. Part 4: Trustworthiness and publishing. Eur. J. Gen. Pract. 2018, 24, 120-124. [CrossRef]

40. De Meester, A.; Aelterman, N.; Cardon, G.; De Bourdeaudhuij, I.; Haerens, L. Extracurricular school-based sports as a motivating vehicle for sports participation in youth: A cross-sectional study. Int. J. Behav. Nutr. Phys. Act. 2014, 11, 48. [CrossRef]

41. Feldman, A.F.; Matjasko, J.L. The Role of School-Based Extracurricular Activities in Adolescent Development: A Comprehensive Review and Future Directions. Rev. Educ. Res. 2005, 75, 159-210. [CrossRef] 
42. Gearing, R.E.; MacKenzie, M.J.; Schwalbe, C.S.; Brewer, K.B.; Ibrahim, R.W. Prevalence of Mental Health and Behavioral Problems Among Adolescents in Institutional Care in Jordan. Psychiatr. Serv. 2013, 64, 196-200. [CrossRef] [PubMed]

43. Schmid, M.; Goldbeck, L.; Nuetzel, J.; Fegert, J.M. Prevalence of mental disorders among adolescents in German youth welfare institutions. Child Adolesc. Psychiatry Ment. Health 2008, 2, 2. [CrossRef] [PubMed]

44. Shulman, E.P.; Cauffman, E. Coping While Incarcerated: A Study of Male Juvenile Offenders. J. Res. Adolesc. 2011, $21,818-826$. [CrossRef] [PubMed] 